\title{
Kardiopulmonale Komorbiditäten
}

Frederik Seiler, Albrecht von Hardenberg, Michael Böhm, Robert Bals, Christoph Maack

Besonders bei älteren Patienten sind kardiopulmonale Komorbiditäten häufig - und die Prognose ist schlecht. Pulmonale Erkrankungen wie z. B. COPD oder die pulmonale Hypertonie beeinflussen das Herz auf vielfältige Weise. Doch die physiologische Achse zwischen linkem Herz, Lunge und rechtem Herz führt auch dazu, dass Herzerkrankungen Lungenprobleme hervorrufen. Die kardiopulmonale Differenzialdiagnostik hat deshalb einen hohen klinischen Stellenwert.

\section{Epidemiologie}

COPD und Herzinsuffizienz | Aufgrund der steigenden Lebenserwartung der Bevölkerung ist die Prävalenz chronischer Erkrankungen erhöht. Zu den häufigsten gehören kardiovaskuläre Erkrankungen, Krebs, Diabetes und chronisch respiratorische Erkrankungen - besonders die chronisch obstruktive Lungenerkrankung (COPD) [1]. Bei COPD liegt in $20 \%$ der Fälle auch eine chronische Herzinsuffizienz vor [2]. Eine reduzierte Einsekundenkapazität $\left(\mathrm{FEV}_{1}\right)$ ist hierbei ein wichtiger unabhängiger Prädiktor für kardiovaskuläre Erkrankungen und Sterblichkeit $[3,4]$.

Ein Viertel der Patienten mit milder bis moderater COPD sterben an den Folgen kardiovaskulärer Erkrankungen [5].

Bei Patienten mit Herzinsuffizienz liegt in 20$32 \%$ der Fälle eine COPD vor [6], die ein unabhängiger Risikofaktor für Tod und Hospitalisierung ist [7]. Ein wesentlicher gemeinsamer Mechanismus, der den Progress sowohl einer COPD als auch der Atherosklerose fördern kann, ist eine chronische systemische Inflammation. Von besonderer Bedeutung sind bei dieser die Zytokine

- Tumornekrosefaktor- $\alpha$ (TNF- $\alpha$ ),

- Interferon- $\gamma($ IFN- $\gamma)$ und

- Interleukin-6 (IL-6) [5].

Weiterhin kommt es bei kardiovaskulären und pulmonalen Erkrankungen auch zu hämodynamischen und neurohumoralen Veränderungen, die Herzmuskel, Gefäße und Lunge beeinträchtigen. Bei Patienten mit dem Leitsymptom Luftnot ist somit die Diagnostik erschwert. Der kardiopulmonalen Differenzialdiagnostik kommt daher ein hoher klinischer Stellenwert zu.

\section{Physiologie und Pathophysiologie}

Hämodynamik I Das linke Herz, die Lunge und das rechte Herz stellen eine physiologische Achse dar, die über den Blutstrom miteinander verknüpft wird ( $\bullet$ Abb. 1). Bei Erkrankungen des linken Ventrikels (LV) erhöht der Rückstau des Blutes in den linken Vorhof und die Lungenstrombahn den pulmonal-kapillären WedgeDruck (PCWP). Geschieht dies akut, so kommt es typischerweise zu einem Lungenödem. Eine chronische LV-Dysfunktion führt zu einer Erhöhung des pulmonal-arteriellen Drucks (PAP) und somit zu pulmonaler Hypertonie (PH) mit Umbauprozessen der Pulmonalarterien (sog. GefäßRemodelling). Bei dieser Form der pulmonalen Hypertonie spricht man von einer postkapillären $\mathrm{PH}$, da ihre Ursache distal des pulmonal-kapillären Gefäßbetts im linken Herzen liegt. Bei kongenitalen Vitien mit Links-Rechts-Shunt führt ein erhöhter pulmonaler Blutfluss zu erhöhtem PAP mit ähnlichen Folgen für Gefäße und rechten Ventrikel $(\mathrm{RV})(\bullet$ Abb. 1).

Abb. 1 Hämodynamische und pathophysiologische Zusammenhänge kardiopulmonaler Komorbiditäten.

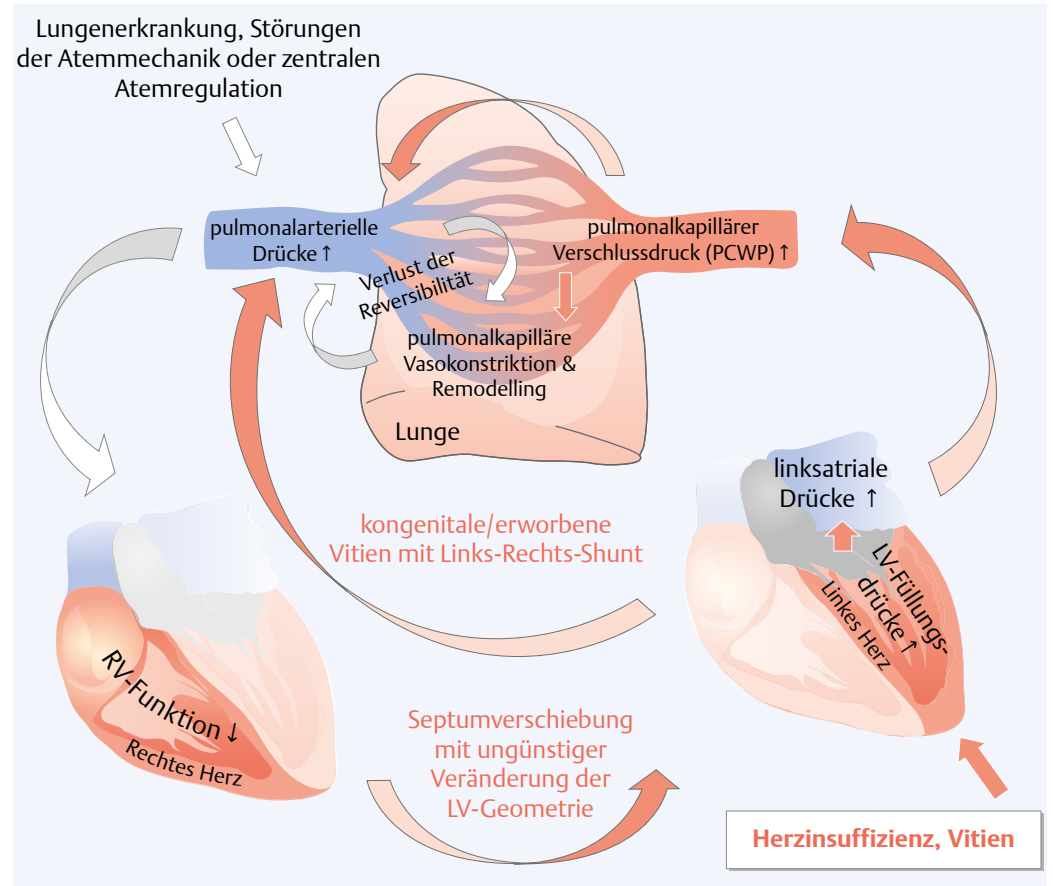

Glossar I

FEV $_{\mathbf{1}}$ :

reduzierte Einsekun-

denkapazität

PCWP:

pulmonal-kapillärer

Wedge-Druck

PAP:

pulmonal-arterieller Druck

PH:

pulmonale Hypertonie 
Glossar II CTEPH:

chronisch thrombembolische $\mathrm{PH}$

PAH:

pulmonal-arterielle Hypertonie
Präkapilläre PH | Alle anderen Formen einer PH ohne Erhöhung des pulmonal-kapillären WedgeDrucks werden als präkapilläre $\mathrm{PH}$ bezeichnet. Erkrankungen der Lunge können die luftleitenden Abschnitte (z. B. bei COPD) und / oder das Parenchym betreffen (z.B. bei Lungenfibrose oder -Emphysem).

- Störungen der Atemmuskulatur (z. B. bei Myasthenia gravis),

- der zentralen Atmungskontrolle (z.B. beim Schlaf-Apnoe-Syndrom),

- massives Übergewicht [16] oder

- dauerhafter Aufenthalt in großen Höhen [17]

führen zu einer alveolären Hypoventilation und dadurch zur hypoxischen pulmonalen Vasokonstriktion (HPV) mit einem Anstieg des PAP ( Abb. 1). Bei parenchymalen Lungenerkrankungen ist ein PAP-Anstieg zusätzlich bedingt durch

- Verlust vaskulärer Oberfläche und / oder

- Complianceverlust durch Lungenüberblähung mit konsekutiver Vasokompression.

RV-Hypertrophie I Der rechte Ventrikel hypertrophiert, um sich an die vermehrte Nachlast bei PH anzupassen. Wenn der RV die vermehrte Arbeit trotz Hypertrophie nicht mehr leisten kann, dilatiert dieser mit kontraktiler Dysfunktion und Stauung des Blutes vor dem RV in

- die Halsvenen,

- die Leber,

- das Integument (Anasarka) und

- die Extremitäten (periphere Ödeme).

Nur eine RV-Dysfunktion, die durch eine isolierte Lungenerkrankung hervorgerufen wird, bezeichnet man als Cor pulmonale $[8,9]$.

Thrombembolien I Bei einer Lungenembolie als Folge venöser Thrombembolien kommt es meist zum schlagartigen Anstieg des pulmonal-arteri-

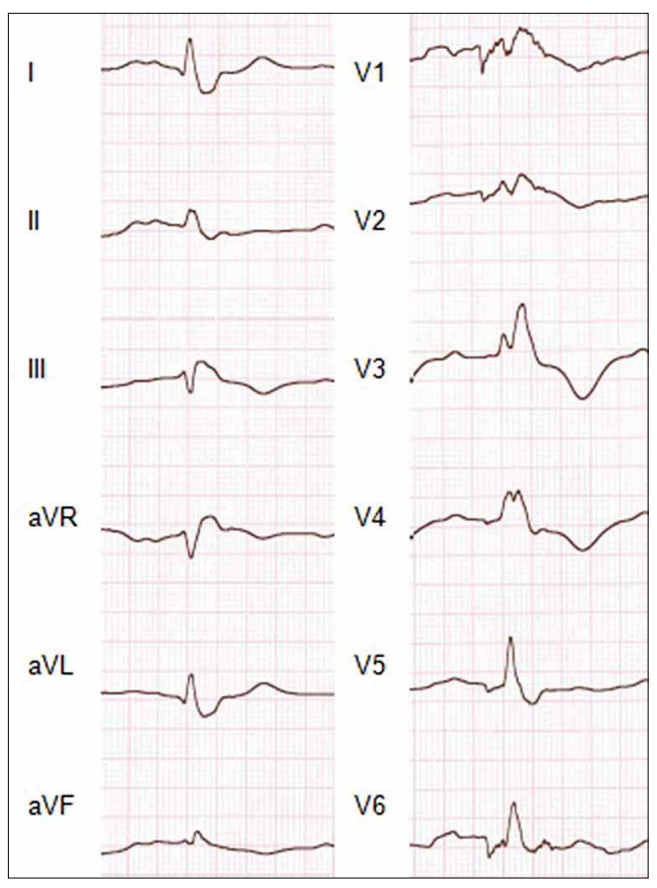

ellen Widerstands. Dies kann bei nicht präkonditioniertem RV zum akuten RV-Versagen führen. Die pulmonale Durchflussfläche vermindern auch - ein Pleuraerguss,

- eine Pneumektomie oder

- ein Pneumothorax.

Eine chronisch thrombembolische PH (CTEPH) entsteht durch erhöhten Gefäßwiderstand. Dieser wird durch einen fibrotischen Einbau von Thromben in die proximalen und intermediären Lungenarterien verursacht. Die idiopathische pulmonal-arterielle Hypertonie (PAH) entsteht durch einen erhöhten Gefäßwiderstand insbesondere auf mikrovaskulärer Ebene.

Alle Formen der pulmonalen Hypertonie führen zu chronischer RV-Belastung mit Hypertrophie und ggf. Insuffizienz. Es ist wichtig, die Ursachen für eine $\mathrm{PH}$ zu diagnostizieren, da hiervon die Therapie abhängt.

Inflammation | Ein wichtiger Risikofaktor für COPD und Atherosklerose ist Tabakrauch. Dieser führt eine pulmonale und systemische Inflammation herbei und inaktiviert über oxidativen Stress Proteasen. Außerdem schädigt er das respiratorische Epithel und führt zu einer Mukus-Hypersekretion - einer Einwanderung von Neutrophilen ins Lungengewebe mit Freisetzung proinflammatorischer Zytokine [10]. Klinisch manifestiert sich dies bei Patienten mit COPD häufig durch

- erhöhtes C-reaktives Protein (CRP),

- Fibrinogen und

- Leukozytose, insbesondere bei Infekt-Exazerbation.

Auch bei der Atherosklerose spielt das Einwandern von T-Lymphozyten mit Sekretion von Zytokinen (IFN- $\gamma$, TNF- $\alpha$, IL-1 und IL-6) eine wichtige Rolle. Weiterhin fördert die Inflammation die Produktion prokoagulatorischer Faktoren (z.B. Fibrinogen). Dies erklärt den engen Zusammenhang zwischen Inflammation und akuten koronaren Syndromen, kardiovaskulärer Morbidität und Sterblichkeit [5].

\section{Kardiopulmonale Differenzialdiagnostik}

Klinische Untersuchung I Für eine rechtskardiale Dysfunktion sprechen

- gestaute Halsvenen mit prominenter a-Welle,

- periphere Ödeme,

- Aszites und

- ein hepatojugulärer Reflux.

Eine zentrale Zyanose, die nach $\mathrm{O}_{2}$-Gabe bestehen bleibt, kann auf einen Rechts-Links-Shunt hinweisen. Trommelschlägelfinger weisen auf eine chronische Hypoxie hin. Bei COPD mit Lungenemphysem bestehen

- Lippenbremse,

- hypersonorer Klopfschall, 
- ein verlängertes Exspirium und

- abgeschwächte Atemgeräusche.

Knistergeräusche und gedämpfter Klopfschall sind typisch für Fibrose. Weiterhin ist bei der Auskultation auf vitientypische Geräusche zu achten. Eine PH kann als betonter oder fixiert gespaltener 2. Herzton womit betontem späten Anteil über dem Pulmonalklappenareal hörbar sein.

EKG | Elektrokardiografische Hinweise für eine akute oder chronische rechtskardiale Belastung sind:

- Zeichen einer RV-Hypertrophie,

- Rechtsherzbelastungszeichen wie $\mathrm{S}_{\mathrm{I}}-\mathrm{Q}_{\mathrm{III}}$ - Typ,

- ein kompletter oder inkompletter Rechtsschenkelblock,

- ein Rechtslagetyp,

- T-Negativierungen in den Brustwand-Ableitungen V2 und V3 oder

- ein p-Pulmonale ( Abb. 2).

Röntgen-Thorax | Eine Erweiterung der Pulmonalarterien und eine periphere Diameterreduktion der Lungengefäße führen häufig zu einem ersten Verdacht auf eine pulmonale Hypertonie. Eine Rechtsherzdilatation füllt in lateraler Projektion den retrosternalen Raum aus ( $\triangle$ Abb. 3). Weiterhin gibt das Röntgenbild wichtige Hinweise auf die zugrunde liegende Erkrankung: z.B. Herzinsuffizienz oder Klappenerkrankungen mit spezifischen Veränderungen des LV oder des Lungenparenchyms. Das Ausmaß einer pulmonalvenösen Stauung kann ebenfalls abgeschätzt werden.

Echokardiografie | Sie erlaubt die Evaluation der - atrialen und ventrikulären Maße,

- systolischen und diastolischen Ventrikel sowie

- Klappenfunktionen.

Der systolische PAP kann abgeschätzt werden, indem man den systolischen Druckgradienten über der Trikuspidalklappe bestimmt. Dies wird ori-

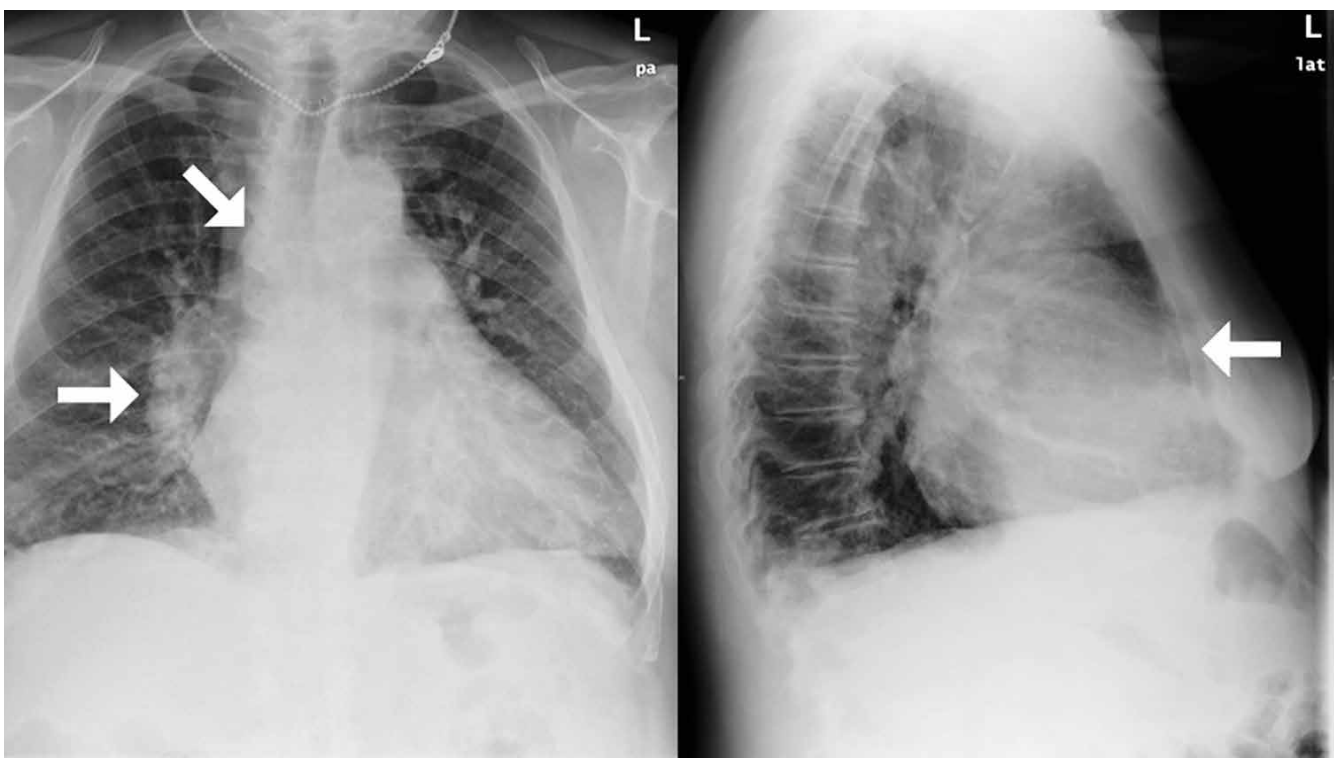

\begin{tabular}{|llll} 
Diagnose & PAPm & PCWP & DGP \\
präkapilläre PH & $\geq 25 \mathrm{mmHg}$ & $\leq 15 \mathrm{mmHg}$ & NN \\
postkapilläre PH & $\geq 25 \mathrm{mmHg}$ & $>15 \mathrm{mmHg}$ & $<7 \mathrm{mmHg}$ \\
kombinierte PH & $\geq 25 \mathrm{mmHg}$ & $>15 \mathrm{mmHg}$ & $\geq 7 \mathrm{mmHg}$
\end{tabular}

entierend zur Differenzialdiagnostik und zur Verlaufsbeurteilung einer PH herangezogen.

Bei pulmonaler Hypertonie ist typischerweise der RV vergrößert und beim Cor pulmonale auch spitzenbildend, was insbesondere im apikalen Vierkammerblick sichtbar wird ( Abb. 4).

Lungenfunktionsdiagnostik | Die Spirometrie und die Bodyplethysmografie identifizieren restriktive und obstruktive Ventilationsstörungen. Die Bestimmung der Diffusionskapazität quantifiziert den pulmonalen Gasaustausch. Eine verringerte Diffusionskapazität ohne relevante Ventilationsstörungen kann auf eine PH hinweisen. Bei Herzinsuffizienz besteht häufig eine restriktive Ventilationsstörung, die bei akuter Dekompensation aufgrund der Lungenstauung um eine obstruktive Komponente erweitert sein kann.

Rechtsherzkatheter | Die Rechtsherzkatheterisierung ist der Goldstandard zur Quantifizierung einer PH und zur Differenzierung in präund/oder postkapilläre Formen. Sie ermöglicht die direkte Bestimmung des pulmonal-arteriellen Drucks und des pulmonal-kapillären WedgeDrucks. In $>$ Tab. 1 sind die diagnostischen Kriterien der PH dargestellt [11-13]. Der mittlere PAP (PAPm) beträgt $14 \pm 3 \mathrm{mmHg}$ bei Gesunden und wird bis $20 \mathrm{mmHg}$ als physiologisch angesehen [13]. Die PH ist definiert als Anstieg des PAPm
Tab. 1 Diagnostische Kriterien der pulmonalen Hypertonie. PAPm: mittlerer pulmonalarterieller Druck; PCWP: pulmonalkapillärer Wedge-Druck; DGP: diastolischer Druckgradient.

Glossar III

PAPm:

mittlerer pulmonal arterieller Druck

Abb. 3 Chronische Rechtsherzbelastung im Röntgen-Thorax eines Patienten mit schwerer IPAH (Idiopathische Pulmonale Hypertonie) und komorbider Linksherzinsuffizienz: (u. a.) dilatierte Pulmonalarterien mit Kalibersprung $(\rightarrow)$, Verengung des Retrosternalraums $(\leftarrow)$ und Erweiterung der $V$. azygos $(\searrow)$. 
Abb. 4 Echokardiografisch apikaler Vierkammerblick bei normalem Herzen (A) und mit ausgeprägter chronischer Rechtsherzbelastung bei einem Patienten mit PH (B).
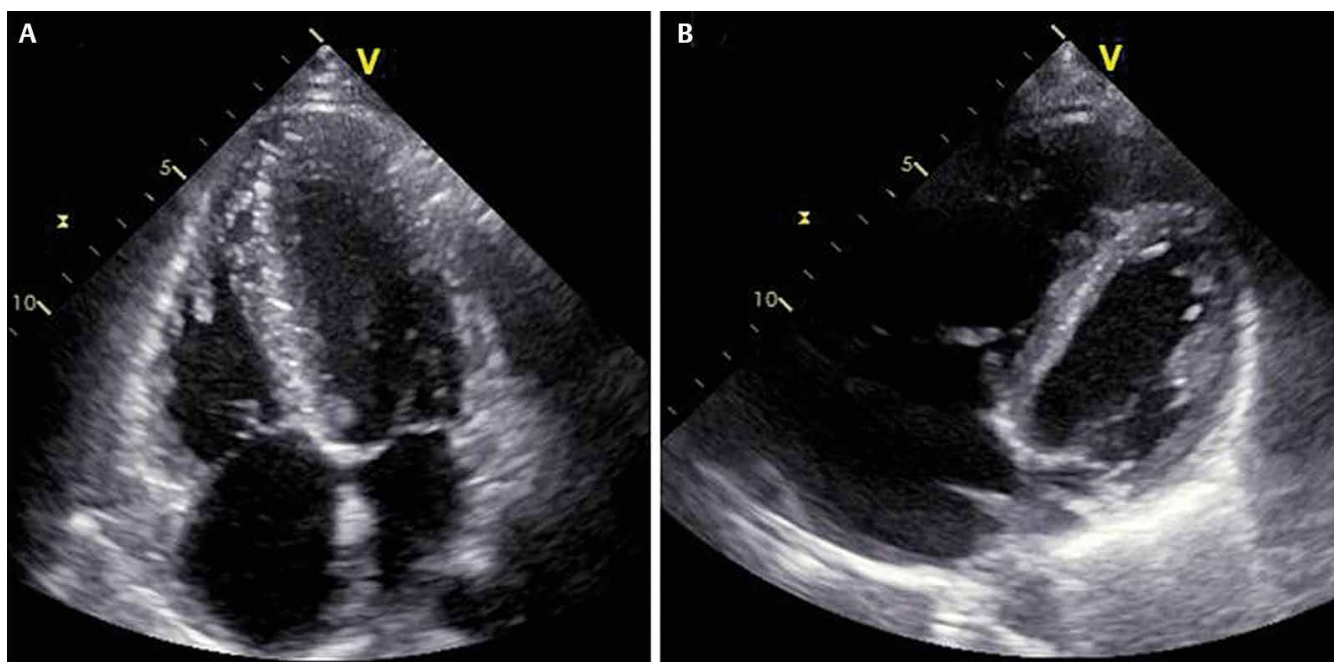

auf $\geq 25 \mathrm{mmHg}$ in Ruhe. Werte von $21-24 \mathrm{mmHg}$ haben unklare klinische Relevanz und sollten regelmäßig kontrolliert werden. Ein pulmonalkapillären Wedge-Druck (PCWP) $>15 \mathrm{mmHg}$ weist auf eine postkapilläre (linkskardiale) Ätiologie der PH hin. Der diastolische Druckgradient (DPG, Differenz aus diastolischem PAP und PCWP) erlaubt eine isolierte postkapilläre von einer kombinierten prä- und postkapillären $\mathrm{PH}$ zu unterscheiden.

Blutgasanalyse I Anhand der arteriellen Blutgasanalyse lassen sich Ausprägung und Charakteristik einer respiratorischen Insuffizienz bestimmen. Die respiratorische Partialinsuffizienz ist gekennzeichnet durch eine Hypoxämie und tritt z.B. bei alveolärer Hypoventilation und Störungen der Diffusion auf (z.B. PH oder interstitielle Lungenerkrankungen). Eine respiratorische Globalinsuffizienz mit Hyperkapnie weist auf ventilatorisches Versagen durch Erschöpfung der Atempumpe hin - wie z.B. bei exazerbierter COPD oder dekompensierter Linksherzinsuffizienz mit Lungenödem.

\section{Welche pulmonalen Erkrankungen beeinflussen das Herz - und wie?}

Hypoxie und Hyperkapnie I Die respiratorische Insuffizienz ist eine typische Konsequenz verschiedener pulmonaler und kardialer Erkrankungen. Sie begünstigt über eine hypoxische pulmonale Vasokonstriktion die Proliferation glatter Gefäßmuskelzellen in der pulmonalen Strombahn. Über Polyglobulie führt sie zum Anstieg des pulmonalvaskulären Widerstands und damit zu einer PH mit Rechtsherzbelastung.

COPD I Bei COPD besteht eine allenfalls teilreversible Atemwegsobstruktion, die im Verlauf zu einer Degeneration der Lungenstruktur und einem progredienten Verlust der Lungenfunktion führt.
Die Koinzidenz von COPD und kardialen Erkrankungen ist häufig: Bei ca. 20\% der COPD-Patienten liegt eine (Links-) Herzinsuffizienz vor und bei $20-31 \%$ der Patienten mit Herzinsuffizienz eine COPD $[5,14]$.

Die COPD ist hierbei ein unabhängiger kardiovaskulärer Risikofaktor und wird insbesondere durch Tabakrauch und andere Umwelt-bedingte inhalative Noxen verursacht. Diese induzieren neben einer pulmonalen auch eine systemische Inflammation, die wiederum Atherosklerose und die Synthese prokoagulatorischer Faktoren begünstigt [5, 14]. Entsprechend ist das Risiko für folgende Erkrankungen bei COPD um das 2- bis 5-Fache erhöht [15]:

- ischämische Herzerkrankung

- Herzrhythmusstörungen

- Herzinsuffizienz

- weitere arterielle Erkrankungen

Das Vorliegen einer PH im Rahmen einer COPD ist ein negativer prognostischer Prädiktor [16]. Die COPD verschlechtert die Prognose bei Patienten trotz der hohen Prävalenz häufig unerkannt [17].

Rauchverzicht bei COPD | Der Rauchverzicht ist die erste und wichtigste therapeutische Maßnahme bei Patienten mit COPD (mit und ohne kardiovaskuläre Komorbidität): Dieser verbessert die Prognose [5]. Allerdings setzt er die Motivation des Patienten voraus und kann neben pharmakologischen auch allgemeine und psychologische Behandlungen umfassen [18]. Therapien, die den Progress kardiovaskulärer Erkrankungen verringern (z.B. ACE-Hemmer/AT1-Antagonisten und Statine), haben auch günstige Effekte auf Morbidität und Prognose einer COPD (mit und ohne kardiovaskuläre Komorbidität) [5, 19].

Betablocker bei COPD? | Die Therapie kardiovaskulärer Erkrankungen mit Betablockern und die Therapie von Patienten mit COPD mit $\beta_{2^{-}}$ mit koronarer Herzkrankheit (KHK), bleibt jedoch 
Mimetika ist ein scheinbares therapeutisches Dilemma. Betablocker galten anfangs aufgrund der möglichen Bronchokonstriktion als relativ kontraindiziert bei COPD.

Neuere Analysen zeigen jedoch eine hohe Sicherheit und sogar günstige funktionelle und prognostische Effekte von $\beta_{1}$-selektiven Betablockern bei COPD mit kardiovaskulären Komorbiditäten.

Zwar kann ein $\beta_{1}$-selektiver Betablocker bei akuter Gabe zu einer passageren, wenn auch unbedeutsamen Verschlechterung der $\mathrm{FEV}_{1}$ um ca. 7\% führen. Die $\mathrm{FEV}_{1}$ ist jedoch bei Langzeittherapie der COPD mit einem $\beta_{1}$-Blocker nicht mehr reduziert [20]. $\beta_{2}$-Mimetika verursachen langfristig eine Tachyphylaxie, ohne die $\mathrm{FEV}_{1}$ zu verbessern [21]. Die akute, aber auch die chronische Gabe eines $\beta_{1}$-Blockers verbessert das Ansprechen der FEV $_{1}$ auf $\beta_{2}$-Mimetika [20] am ehesten, weil $\beta_{2}$ Adrenozeptoren resensibilisiert werden [22]. Bei obstruktiven Lungenerkrankungen hält die Aktivierung von $\beta_{2}$-Adrenozeptoren eine pulmonale Inflammation mit muköser Metaplasie und hyperreagiblem Bronchialsystem aufrecht. Deshalb können Betablocker die pulmonale Inflammation und den Phänotyp langfristig verbessern [23]. Bei Patienten nach Herzinfarkt ist bei einer COPD die absolute Risikoreduktion durch Betablocker größer als bei Patienten ohne COPD (bei gleicher relativer Risikoreduktion) [24]. Im Gegensatz dazu erhöhen $\beta_{2}$-Mimetika bei COPD-Behandlung Arrhythmien und andere kardiovaskuläre Ereignisse [25].

Daher sollten Patienten mit kardiovaskulären Erkrankungen (insbes. Herzinsuffizienz) und COPD Betablocker nicht vorenthalten werden. Lediglich $\beta_{2}$-Mimetika sollten so weit wie möglich vermieden werden.

Idiopathische Lungenfibrose I Die idiopathische Lungenfibrose (IPF) ist die häufigste Form der idiopathischen interstitiellen Pneumonien. Die Prognose ist schlecht und die Therapieoptionen limitiert. Obwohl die häufigste Todesursache bei Patienten mit IPF das respiratorische Versagen ist, scheinen auch hier kardiale Komorbiditäten wesentlich zur Prognose beizutragen [26]. IPF-Patienten haben ein erhöhtes kardiovaskuläres Risiko im Vergleich zur Normalbevölkerung [27] - etwa ein Viertel der Todesfälle fällt auf kardiale Ursachen zurück. Insbesondere die Häufigkeit einer KHK ist erhöht [28, 29]. Die histopathologischen Merkmale der IPF sind vergleichbar mit denen der Atherosklerose [30]. Es wird vermutet, dass durch systemische fibroproliferative Effekte der IPF begünstigt eine Atherosklerose entsteht und fortschreitet [29]. Ähnlich wie bei der COPD ist

auch bei IPF eine gleichzeitige PH mit einer schlechten Prognose assoziiert [26, 31].

Lungenembolie | Die Lungenembolie ist eine Manifestation der venösen Thrombembolie, bei der Abschnitte der Lungenstrombahn durch thrombotisches Material verlegt werden. Dadurch nimmt der Gefäßabschnitt, der distal der Okklusion liegt, nicht mehr am Gasaustausch teil - die Oxygenierung wird verschlechtert.

Bedeutsamer als die respiratorische Insuffizienz ist jedoch der abrupte Anstieg des pulmonalvaskulären Widerstands.

Dieser erhöht akut die Nachlast des RV. Bei nichtpräkonditionierten Patienten kommt es bereits bei einer Verlegung von ca. 50\% der Lungenstrombahn oder einem Anstieg des PAPm auf ca. $40 \mathrm{mmHg} z u$

- einem Abfall der Auswurfleistung,

- einem weiteren Druckanstieg und

- letztlich zum akuten Rechtsherzversagen.

Das plötzlich erhöhte RV-Volumen komprimiert zudem den LV und beeinträchtigt somit dessen diastolische Funktion [32, 33]. Je nach Ausprägung der Embolisation umfasst die Lungenembolie eine Bandbreite von subklinischen Verläufen bis hin zum plötzlichen Herztod. Die Risikostratifizierung beinhaltet insbesondere echokardiografische und laborchemische Zeichen der RVBelastung ( Tab. 2).

Pulmonale Hypertonie: Klassifikation | Die pulmonale Hypertonie wird in 5 Gruppen und mehrere Untergruppen unterteilt $(\triangleright$ Tab. 3 ). Die häufigsten Formen sind die

- PH bei Linksherzerkrankungen (Gruppe 2), bei denen eine postkapilläre PH vorliegt und

- PH bei Lungenerkrankungen und/oder Hypoxie (Gruppe 3).

In Gruppe 1 gehören Patienten mit pulmonal-arterieller Hypertonie (PAH; Prävalenz: ca. 15-60 Fälle pro 1 Mio. Einwohner in Europa). In dieser Gruppe ist eine Hypertrophie und somit Versteifung der Pulmonalarterien die grundlegende Ursache. Die chronisch-thromboembolische $\mathrm{PH}$

$\begin{array}{llll}\text { Diagnose } & \text { hohes Risiko } & \text { intermediä- } & \text { niedriges } \\ & \text { (Letalität>15\%) } & \text { res Risiko } & \text { Risiko } \\ & & \text { (Letalität <1\%) }\end{array}$

hämodynamische

Instabilität

echokardiografische

Zeichen der RV-Dys-

funktion

laborchemische

Zeichen der myokardia-

len Schädigung
Glossar IV

IPF:

idiopathische Lungen-

fibrose
Tab. 2 Risikostratifizierung der Lungenarterienembolie. 


\section{Nizza-Klassifikation: pulmonale Hypertonie}

$\begin{array}{lll}\text { Gruppe } 1 & \begin{array}{l}\text { Pulmonal-arterielle } \\ \text { Hypertonie (PAH) }\end{array} & \begin{array}{l}\text { umfasst u. a.: } \\ \text { Idiopathische Pulmonale Hypertonie } \\ \text { (IPAH) }\end{array} \\ \text { Gruppe } 2 & \begin{array}{l}\text { PH bei Linksherz- } \\ \text { erkrankungen }\end{array} & \text { (APAH), z. B. bei Kollagenosen } \\ & & \text { umfast u. a.: }\end{array}$

Tab. 3 Vereinfachte Nizza-Klassifikation der Pulmonalen Hypertonie.
(CTEPH) ist mit ca. 3 Fällen pro 1 Mio Einwohner noch seltener. In etwa 75\% der Fälle ist sie Folge einer oder mehrerer Lungenembolien. Die Gruppe 5 besteht aus Patienten mit PH mit unklarer und / oder multifaktorieller Genese [13].

Therapie der pulmonal arteriellen Hypertonie| Ziel ist es, den pulmonalvaskulären Widerstand zu senken und damit den RV zu entlasten. Bei der pulmonal-arteriellen Hypertonie (PH Klasse 1) kommen spezifische medikamentöse Therapien zum Einsatz [11, 34]: Hemmstoffe der Phosphodiesterase 5 (PDE5-Hemmer) wie Sildenafil und Tadalafil erhöhen die Konzentration von cGMP und lockern die glatten Gefäßmuskelzellen - es kommt zu einer Vasodilatation. Über den gleichen Signalweg wirken auch der lösliche Guanylatzyklase-Aktivator Riociguat und inhalatives Stickstoffmonoxid. Folgende Endothelin-Rezeptor Antagonisten hemmen die vasokonstriktorischen und profibrotischen Wirkungen von Endothelin an der glatten Gefäßmuskulatur:

- Bosentan

- Ambrisentan

- Macitentan

Prostazyklin-Analoga (z.B. Epoprostenol, Iloprost, Treprostinil und Beraprost) werden überwiegend intravenös, inhalativ oder subkutan appliziert. Einzig für Treprostinil steht eine orale Formulierung zur Verfügung. Die meisten Substanzen verbessern die Symptomatik. Selexipag ist ein oral verfügbarer selektiver Prostazyklin-IPRezeptor-Agonist. Dieser verbesserte als Monotherapie oder in Kombination mit einem Endo-
thelin-Rezeptor-Antagonisten und/oder einem PDE5-Hemmer die Symptome und reduzierte auch das Auftreten eines kombinierten Morbiditäts- und Mortalitäts-Endpunkts [13].

Cave Die PAH ist seltener als PH-Formen der Gruppen 2 und 3. Somit besteht nur für einen geringen Teil der Patienten eine spezifische medikamentöse Therapie.

Therapie der CTEPH | Bei der chronisch-thromboembolischen pulmonalen Hypertonie (CTEPH) besteht eine prinzipiell kurative chirurgische Therapieoption: die pulmonale Thrombendarteriektomie. Patienten mit CTEPH sollten daher frühzeitig in einem entsprechenden Expertenzentrum vorgestellt werden. Der Eingriff ist mit einer perioperativen Mortalität von ca. 5\% assoziiert [35]. Eine medikamentöse Therapie mit Riociguat ist nur bei Patienten mit Kontraindikationen für eine Operation oder bei persistierender $\mathrm{PH}$ nach pulmonaler Thrombendarteriektomie zugelassen [13]. Zudem ist eine therapeutische Antikoagulation indiziert [36].

Therapie der PH bei chronischen Lungenerkrankungen | Bei den übrigen Formen der präkapillären PH (insbes. der Gruppe 3) steht die Therapie der Grunderkrankung im Vordergrund. Bei respiratorischer Insuffizienz ist eine Langzeit-Sauerstofftherapie indiziert, um die hypoxische pulmonale Vasokonstriktion zu verringern und die Lebensqualität zu verbessern. Bei PH im Rahmen einer COPD senkt die Langzeit-Sauerstofftherapie auch die Sterblichkeit [16]. Der Nutzen einer medikamentös-vasodilatatorischen Therapie bei einer PH der Gruppe 3 ist unklar und sollte nur in Expertenzentren erfolgen [13]. Hierbei handelt es sich um einen Off-label-Einsatz.

Therapie der PH bei Linksherzerkrankungen I Behandelt wird die Herzerkrankung. Die bei der Linksherzinsuffizienz etablierten Therapiekonzepte (z.B. ACE-Hemmer, Betablocker, Aldosteron-Antagonisten) haben in der Therapie der Rechtsherzinsuffizienz bei PH keinen nachgewiesenen Stellenwert [16, 37]. Die Ultima Ratio bei allen Formen der PH und geeigneten Patienten ist die Lungentransplantation bzw. bei terminaler RV-Dysfunktion die Herz-Lungentransplantation.

Schlafbezogene Atmungsstörungen I Kardiale Komorbiditäten treten bei diesen Patienten gehäuft auf: Zum einen überschneiden sich die Risikofaktoren wie z.B. Alter, männliches Geschlecht und Adipositas. Ein weiterer Grund sind die pathophysiologischen Vorgänge bei schlafbezogenen Atmungsstörungen. Charakteristisch für diese Erkrankungen ist ein ständiger Wechsel zwischen Hypoxie und Reoxygenierung. Dieser resultiert über oxidativen Stress in einer sympa- 
thikoadrenergen Aktivierung mit erhöhter endogener Katecholaminfreisetzung und systemischer Inflammation. Begünstigt werden dadurch:

- eine arterielle Hypertonie

- myokardiale Remodelingprozesse

- eine endotheliale Dysfunktion

- Atherosklerose

Daher haben Patienten mit schlafbezogenen Atmungsstörungen ein erhöhtes Risiko für Herzrhythmusstörungen (z. B. Vorhofflimmern), KHK und chronische Herzinsuffizienz [38].

Eine sich im Rahmen der chronischen Hypoxie entwickelnde PH kann zudem im Verlauf zur RVBelastung führen. Dieser Verlauf ist jedoch bei einem klassischen Schlafapnoesyndrom ohne tagsüber bestehende Hypoxie und pulmonale Komorbidität eher untypisch [39].

Obstruktive und zentrale Schlaf-Apnoe I Man unterscheidet das obstruktive (OSA) und das zentrale Schlaf-Apnoe-Syndrom (CSA). Das OSA tritt typischerweise bei adipösen Patienten auf: Im Schlaf erschlafft die Muskulatur und der Zungengrund verlegt die oberen Luftwege. Alkohol kann dies noch verstärken. Bei Obstruktion der oberen Atemwege kommt es zu frustranen Atemmanövern, die einen negativen intrathorakalen Druck provozieren. Im Gegensatz dazu kommt es beim CSA zu einer zentral vermittelten Cheyne-StokesAtmung mit Phasen von Apnoe oder Hypopnoe im Wechsel mit Hyperventilation.

Das CSA ist mit einer Prävalenz von 30-50\% eine häufige Komorbidität der chronischen Herzinsuffizienz und ein negativer prognostischer Prädiktor [40].

Therapie des OSA | Als Therapie von OSA und CSA stehen verschiedene Formen der nichtinvasiven Beatmung zur Verfügung. Die häufigste ist die CPAP-Beatmung: Bei dieser wird konstanter positiver end-exspiratorischer Druck (PEEP) über eine Gesichts- oder Nasenmaske appliziert. Bei OSA-Patienten ist die CPAP-Beatmung effektiv und die primäre Behandlungsoption [40].

Therapie des CSA IDie CANPAP-Studie (Canadian Positive Airway Pressure Trial for Heart Failure Patients with Central Sleep Apnea) untersuchte die Effektivität der CPAP-Beatmung bei zentralem Schlaf-Apnoe-Syndrom und Herzinsuffizienz [41]: Der primäre Endpunkt transplantationsfreies Überleben wurde in der Gesamtanalyse nicht erreicht. Doch zeigte eine Posthoc-Analyse, dass bei den Patienten der primäre Endpunkt gesenkt wurde, bei denen CPAP den Apnoe-Hypopnoe-Index auf Werte $<15$ reduzierte ( $57 \%$ der Patienten) [42]. Die Adaptive Druckunterstützungs-Servo-
Ventilation appliziert neben einem positiven end-exspiratorischen Druck bei Hypopnoe- oder Apnoe-Phasen auch kontrollierte Atemzüge. Das soll zyklische Anstiege des $\mathrm{CO}_{2}$ und Hypoxien vermeiden. In der aktuellen SERVE-HF-Studie verbesserte dies bei Patienten mit systolischer Herzinsuffizienz jedoch nicht die Symptomatik oder Lebensqualität - die kardiovaskuläre und Gesamtsterblichkeit stiegen sogar an [43]

Die Adaptive Druckunterstützungs-ServoVentilation kann bei systolischer Herzinsuffizienz derzeit nicht empfohlen werden.

\section{Welche kardialen Erkrankungen beeinflussen die Lunge - und wie?}

PH bei Linksherzerkrankungen I Linkskardiale Erkrankungen sind die häufigste Ursache einer PH. Bei $65-80 \%$ der Patienten mit PH sind linkskardiale Erkrankungen die Ursache:

- die Herzinsuffizienz mit erhaltener (HFpEF) oder mit

- reduzierter Ejektionsfraktion (HFrEF) und

- Klappenvitien ( Abb. 1) [44]

Die erhöhten LV-Füllungsdrücke führen über eine Druckerhöhung in der Lungenstrombahn zu einer Intima-Proliferation der pulmonalen Widerstandsgefäße. Dieses Remodeling kann nach Behandlung der kardialen Grunderkrankung reversibel sein - es kann aber auch in unterschiedlichem Ausmaß irreversibel bleiben („Fixation“ der PH). Als Parameter und prognostischer Marker der linkskardialen Erkrankung und der postkapillären PH dient die RV-Funktion [45-50]. Somit kann man eine PH und deren Ausmaß auch zur Risikostratifizierung und Therapieentscheidung bei Patienten mit Vitien berücksichtigen [48, 51-53].

Systolische und diastolische Herzinsuffizienz I Etwa die Hälfte der Patienten mit Herzinsuffizienz weisen eine erhaltene systolische Funktion auf (LVEF $>=50 \%$; HFpEF). Bei der anderen Hälfte ist die systolische Funktion eingeschränkt $(\mathrm{EF}<50 \%$; HFrEF). Sowohl in der Folge der systolischen als auch der diastolischen Herzinsuffizienz kann sich eine postkapilläre PH entwickeln. Dies ist generell mit dem Schweregrad der Erkrankung und einer schlechteren Prognose assoziiert. Die Prognose der Herzinsuffizienz mit erhaltener Ejektionsfraktion ist generell etwas besser als die mit reduzierter Ejektionsfraktion [54]. Allerdings betrifft die HFpEF eher ältere und weibliche Patienten mit mehr Komorbiditäten - dadurch sind sowohl Sterblichkeit als auch Morbidität durch nicht-kardiale Ursachen zusätzlich kompromittiert. Insbesondere bei HFpEF sind die Prävalenz und der Schweregrad einer PH hoch [55].

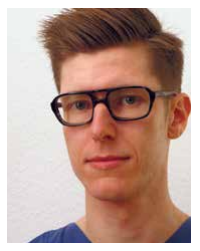

Dr. med. Frederik Seiler ist Assistenzarzt an der Klinik für Innere Medizin V am Universitätsklinikum des Saarlandes. Frederik.Seiler@uniklinikumsaarland.de

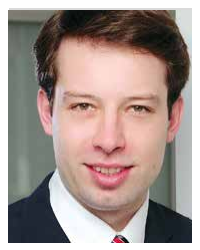

Albrecht von Hardenberg ist ärztlicher Mitarbeiter an der Klinik für Innere Medizin III am Universitätsklinikum des Saarlandes.

Albrecht.Hardenberg@ uniklinikum-saarland.de

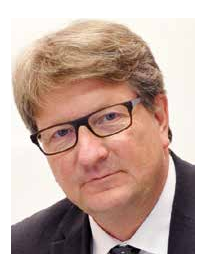

Univ.-Prof. Dr. med. Michael Böhm ist Direktor der Klinik für Innere Medizin III am Universitätsklinikum des Saarlandes.

Michael.Boehm@

uniklinikum-saarland.de

\section{Glossar V}

OSA:

obstruktives Schlaf-

Apnoe-Syndrom

CSA:

zentrales Schlaf-Apnoe-

Syndrom

HFpEF:

Herzinsuffizienz mit erhaltener Ejektionsfraktion

HFrEF:

Herzinsuffizienz mit reduzierter Ejektionsfraktion 


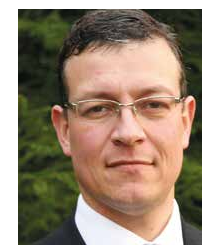

Univ.-Prof. Dr. med. Dr. rer. nat. Robert Bals ist Direktor der Klinik für Innere Medizin V am Universitätsklinikum des Saarlandes.

robert.bals@uks.eu

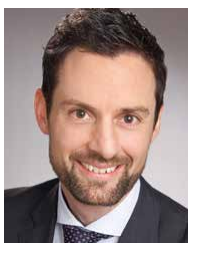

Univ.-Prof. Dr. med. Christoph Maack

ist Oberarzt an der Klinik für Innere Medizin III am Universitätsklinikum des Saarlandes. christoph.maack@uks.eu

\author{
Glossar VI \\ MI: \\ Mitralinsuffizienz
}

DOI 10.1055/s-0041-108687 VNR 2760512016149753950 Dtsch Med Wochenschr 2016; 141: 275-284 (c) Georg Thieme Verlag KG . Stuttgart · New York . ISSN 0012-0472
Zur Zeit besteht keine Evidenz für den Einsatz von Substanzen, die den pulmonalvaskulären Widerstand bei Patienten mit Herzinsuffizienz gezielt senken [56].

Es steht vielmehr die Leitlinien-gerechte Therapie der HFrEF im Vordergrund (ACE-Hemmer, AT1Antagonisten, Betablocker, Aldosteron-Antagonisten, Ivabradin, Diuretika etc.). Bei Patienten mit HFpEF ist eine Therapie der Risikofaktoren indiziert (Diabetes, Hypertonie, Adipositas) [57]. Ausdauersport beeinflusst die Symptomatik sowohl bei Patienten mit HFpEF als auch HFrEF günstig.

Vitien I Die PH ist eine typische Folge von Vitien der Aorten- oder Mitralklappe: Sie tritt bei 15$60 \%$ aller Patienten mit symptomatischen Vitien auf und ist mit einem höheren Risiko für kardiale Ereignisse unter konservativer, operativer oder interventioneller Therapie assoziiert.

Mitralinsuffizienz (MI) I Liegt eine strukturelle oder degenerative Pathologie der Mitralklappe zugrunde, wird die Mitralinsuffizienz als primär oder organisch klassifiziert. Von sekundärer oder funktioneller MI spricht man in Abwesenheit einer organischen Erkrankung der Klappe. In diesem Fall ist die Klappe aufgrund einer LV-Dilatation und / oder -Dysfunktion erkrankt. Die sekundäre MI ist häufiger und hat aufgrund der meist vorliegenden Kardiomyopathie die schlechtere Prognose [58].

Behandlung der MI I Therapeutisch kommt neben operativer Mitralklappen-Rekonstruktion oder -Ersatz das interventionelle Mitra-Clipping zum Einsatz. Grundpfeiler der Behandlung der LV-Dysfunktion ist die leitliniengerechte Herzinsuffizienztherapie: Dies-e beinhaltet neben

- der medikamentösen Therapie bei Vorliegen eines Linksschenkelblocks (QRS > 120 ms) auch

- eine kardiale Resynchronisations-Therapie (CRT) und

- bei Vorliegen einer ischämischen Genese eine wenn möglich komplette myokardiale Revaskularisation [57].

Verbessert sich die Hämodynamik einer PH nach Mitra-Clipping, ist dies bei Patienten mit Herzinsuffizienz mit einer günstigen Prognose assoziiert [59]. Reduziert eine kardiale Resynchronisations-Therapie die MI, kann dies die Hospitalisierung verringern und das Überleben verlängern [60].

Aortenstenose | Die Komorbidität von PH und Aortenstenose ist assoziiert mit

- eingeschränkter LV-Funktion,

- einer Mitralinsuffizienz,

- einer LV-Dilatation und

- erhöhten LV-Füllungsdrücken [61].
Der Grad der PH hängt hierbei hauptsächlich von der diastolischen und weniger der systolischen Dysfunktion oder der Schwere der Aortenstenose ab. Ein erhöhter PAP ist ein Marker für eine schlechte Prognose bei Patienten mit Aortenstenose. Die Abnahme des PAP nach operativem oder interventionellem Aortenklappenersatz zeigt dagegen eine gute Prognose an $[62,63]$.

Mitralstenose und Aorteninsuffizienz | Patienten mit einer Mitralstenose und einer schweren $\mathrm{PH}$ haben eine höhere NYHA-Klasse vor oder nach Valvuloplastie als Patienten ohne PH [64]. Ihre Lebenserwartung liegt bei weniger als 3 Jahren [65]. Die dominierenden Todesursachen sind

- kongestive Herzinsuffizienz,

- akutes Lungenödem und

- Rechtsherzversagen.

Zur PH in Assoziation mit einer Aorteninsuffizienz ist nur wenig bekannt. Hierbei werden zur Risikostratifizierung und Therapieentscheidung eher LV-Funktion und -Dimension sowie die klinische Symptomatik zugrunde gelegt. Das Vorliegen einer PH fließt nicht zwingend in die Entscheidung mit ein [66].

\section{Konsequenz für Klinik und Praxis}

- Kardiopulmonale Komorbiditäten sind häufig, treten insbesondere in der alternden Bevölkerung auf und haben eine schlechte Prognose.

- Insbesondere eine systemische Inflammation - u. a. durch Tabakrauch - bedingt die Komorbidität von COPD und Atherosklerose.

- Die Ursachen für eine pulmonale Hypertonie sind vielschichtig und die Therapien gegen die zugrundeliegenden Ursachen gerichtet. Die häufigste Ursache ist eine Linksherzinsuffizienz.

- Eine umfassende und zielgerichtete Differenzialdiagnostik in einem Expertenzentrum ist eine wichtige Voraussetzung für eine adäquate Therapie kardiopulmonaler Komorbiditäten.

- Patienten mit Herzinsuffizienz und/oder abgelaufenem Myokardinfarkt sollten auch bei einer COPD Betablocker erhalten.

- Bei COPD-Patienten sollte die Therapie mit $\beta_{2}$-Mimetika bei kardiovaskulären Komorbiditäten soweit wie möglich vermieden werden.

\section{Interessenkonflikt}

Die Autoren geben an, dass kein Interessenkonflikt besteht.

Vollständiges Literaturverzeichnis unter http://dx.doi.org/10.1055/s-0041-108687 
Literatur

1 Horton R. The neglected epidemic of chronic disease. Lancet 2005; 366: 1514

2 Rutten FH, Cramer MJ, Lammers JW et al. Heart failure and chronic obstructive pulmonary disease: An ignored combination? Eur J Heart Fail 2006; 8: 706-711

3 Young RP, Hopkins R, Eaton TE. Forced expiratory volume in one second: not just a lung function test but a marker of premature death from all causes. Eur Respir ] 2007; 30: 616-622

4 Sin DD, Wu L, Man SF. The relationship between reduced lung function and cardiovascular mortality: a population-based study and a systematic review of the literature. Chest 2005; 127: 1952-1959

5 Ukena C, Mahfoud F, Kindermann M et al. The cardiopulmonary continuum systemic inflammation as ,common soil ' of heart and lung disease. Int ] Cardiol 2010; 145: 172-176

6 Le Jemtel TH, Padeletti M, Jelic S. Diagnostic and therapeutic challenges in patients with coexistent chronic obstructive pulmonary disease and chronic heart failure.] Am Coll Cardiol 2007; 49: 171-180

7 Hawkins NM, Petrie MC, Jhund PS et al. Heart failure and chronic obstructive pulmonary disease: diagnostic pitfalls and epidemiology. Eur ] Heart Fail 2009; 11: 130-139

8 World Health Organization. Chronic cor pulmonale. Report of an expert committee. World Health Organ Tech Rep Ser 1961; 213: 35

9 Budev MM, Arroliga AC, Wiedemann HP, Matthay RA. Cor pulmonale: an overview. Semin Respir Crit Care Med 2003; 24: 233-244

10 Fabbri LM, Luppi F, Beghe B, Rabe KF. Complex chronic comorbidities of COPD. Eur Respir J 2008; 31: 204-212

11 Galiè N, Hoeper MM, Humbert M et al. Guidelines for the diagnosis and treatment of pulmonary hypertension: the Task Force for the Diagnosis and Treatment of Pulmonary Hypertension of the European Society of Cardiology (ESC) and the European Respiratory Society (ERS), endorsed by the International Society of Heart and Lung Transplantation (ISHLT). Eur Heart ] 2009; 30: 2493-2537

12 Vachiery J-L, Adir Y, Barbera JA et al. Pulmonary hypertension due to left heart diseases. J Am Coll Cardiol 2013; 62: D100-D108

13 Galie N, Humbert M, Vachiery JL et al. 2015 ESC/ERS Guidelines for the diagnosis and treatment of pulmonary hypertension: The Joint Task Force for the Diagnosis and Treatment of Pulmonary Hypertension of the European Society of Cardiology (ESC) and the European Respiratory Society (ERS)Endorsed by: Association for European Paediatric and Congenital Cardiology (AEPC), International Society for Heart and Lung Transplantation (ISHLT). Eur Heart ] 2015; DOI: 10. 1093/ eurheartj/ ehv317

14 Güder G, Rutten FH. Comorbidity of heart failure and chronic obstructive pulmonary disease: more than coincidence. Curr Heart Fail Rep 2014; 11 : 337-346

15 Chen W, Thomas J, Sadatsafavi M, FitzGerald JM. Risk of cardiovascular comorbidity in patients with chronic obstructive pulmonary disease: a systematic review and meta-analysis. Lancet Resp Med 2015; 3: 631-639

16 Zangiabadi A, De Pasquale CG, Sajkov D. Pulmonary hypertension and right heart dysfunction in chronic lung disease. Biomed Res Int 2014; 2014: 739674

17 Almagro P, Lapuente A, Pareja J et al. Underdiagnosis and prognosis of chronic obstructive pulmonary disease after percutaneous coronary intervention: a prospective study. Int ] Chron Obstruct Pulmon Dis 2015; 10: 1353-1361

18 Fiore MC, Baker TB. Clinical practice. Treating smokers in the health care setting. New Engl ] Med 2011; 365: 1222-1231

19 Mancini GB, Etminan M, Zhang B et al. Reduction of morbidity and mortality by statins, angiotensinconverting enzyme inhibitors, and angiotensin receptor blockers in patients with chronic obstructive pulmonary disease. I Am Coll Cardiol 2006; 47: 2554-2560

20 Salpeter SR, Ormiston TM, Salpeter EE. Cardioselective beta-blockers in patients with reactive airway disease: a meta-analysis. Ann Int Med 2002; 137: 715-725

21 Salpeter SR, Ormiston TM, Salpeter EE. Metaanalysis: respiratory tolerance to regular beta2agonist use in patients with asthma. Ann Int Med 2004; 140: 802-813

22 Callaerts-Vegh Z, Evans KL, Dudekula N et al. Effects of acute and chronic administration of beta-adrenoceptor ligands on airway function in a murine model of asthma. Proc Natl Acad Sci USA 2004; 101: 4948-4953

23 Nguyen LP, Lin R, Parra S et al. Beta2-adrenoceptor signaling is required for the development of an asthma phenotype in a murine model. Proc Natl Acad Sci USA 2009; 106: 2435-2440

24 Gottlieb SS, McCarter RJ, Vogel RA. Effect of beta-blockade on mortality among high-risk and low-risk patients after myocardial infarction. New Engl J Med 1998; 339: 489-497

25 Salpeter SR, Ormiston TM, Salpeter EE. Cardiovascular effects of beta-agonists in patients with asthma and COPD: a meta-analysis. Chest 2004; 125: 2309-2321

26 Daniels CE, Yi ES, Ryu JH. Autopsy findings in 42 consecutive patients with idiopathic pulmonary fibrosis. Eur Resp J 2008; 32: 170-174

27 Hubbard RB, Smith C, Le Jeune I et al. The association between idiopathic pulmonary fibrosis and vascular disease. Am J Respir Crit Care Med 2008; 178: 1257-1261

28 Izbicki G, Ben-Dor I, Shitrit D et al. The prevalence of coronary artery disease in end-stage pulmonary disease: is pulmonary fibrosis a risk factor? Resp Med 2009; 103: 1346-1349

29 Kizer JR, Zisman DA, Blumenthal NP et al. Association between pulmonary fibrosis and coronary artery disease. Arch Intern Med 2004; 164: 551-556

30 Epstein FH , Ross R. Atherosclerosis - an inflammatory disease. New Engl ] Med 1999; 340: 115-126

31 Lettieri CJ. Prevalence and outcomes of pulmonary arterial hypertension in advanced idiopathic pulmonary fibrosis. CHEST 2006; 129: 746-752

32 Tapson VF. Acute pulmonary embolism. New Engl ] Med 2008; 358: 1037-1052

33 Matthews JC, McLaughlin V. Acute right ventricular failure in the setting of acute pulmonary embolism or chronic pulmonary hypertension: a detailed review of the pathophysiology, diagnosis, and management. Curr Cardiol Rev 2008; 4: 49

34 Humbert M, Ghofrani H-A. The molecular targets of approved treatments for pulmonary arterial hypertension. Thorax 2015; DOI: 10.1136/ thoraxjnl-2015-207170

35 Jenkins D. Pulmonary endarterectomy: the potentially curative treatment for patients with chronic thromboembolic pulmonary hypertension. Eur Resp Rev 2015; 24: 263-271

36 Hoeper MM. Pharmacological therapy for patients with chronic thromboembolic pulmonary hypertension. Eur Resp Rev 2015; 24: 272-282 
37 Handoko ML, de Man FS, Allaart CP et al. Perspectives on novel therapeutic strategies for right heart failure in pulmonary arterial hypertension: lessons from the left heart. Eur Resp Rev 2010; 19: 72-82

38 Lévy P, Ryan S, Oldenburg O, Parati G. Sleep apnoea and the heart. Eur Resp Rev 2013; 22: 333-352

39 Hawryłkiewicz I, Sliwiński P, Górecka D et al. Pulmonary haemodynamics in patients with OSAS or an overlap syndrome. Monaldi Arch Chest Dis 2004; 61: 148-152

40 Costanzo MR, Khayat R, Ponikowski P et al. Mechanisms and clinical consequences of untreated central sleep apnea in heart failure. J Am Coll Cardiol 2015; 65: 72-84

41 Bradley TD, Logan AG, Kimoff RJ et al. Continuous positive airway pressure for central sleep apnea and heart failure. N Engl J Med 2005; 353: 2025-2033

42 Arzt M, Floras JS, Logan AG et al. Suppression of central sleep apnea by continuous positive airway pressure and transplant-free survival in heart failure: a post hoc analysis of the Canadian Continuous Positive Airway Pressure for Patients with Central Sleep Apnea and Heart Failure Trial (CANPAP). Circulation 2007; 115: 3173-3180

43 Cowie MR, Woehrle H, Wegscheider K et al. Adaptive servo-ventilation for central sleep apnea in systolic heart failure. New Engl J Med 2015; 373 : 1095-1105

44 Held M, Rosenkranz S. Pulmonary hypertension with lung and left-sided heart diseases. Pneumologe $2015 ; 12: 410-416$

45 Park S], Park J-H, Lee HS et al. Impaired RV global longitudinal strain is associated with poor long-term clinical outcomes in patients with acute inferior STEMI. JACC Cardiovasc Imaging 2015; 8: 161-169

46 Ghio S, Gavazzi A, Campana C et al. Independent and additive prognostic value of right ventricular systolic function and pulmonary artery pressure in patients with chronic heart failure. I Am Coll Cardiol 2001; 37: 183-188

47 Melenovsky V, Hwang SJ, Lin G et al. Right heart dysfunction in heart failure with preserved ejection fraction. Eur Heart ] 2014; 35: 3452-3462

48 Magne J, Pibarot P, Sengupta PP et al. Pulmonary hypertension in valvular disease. JACC Cardiovasc Imaging 2015; 8: 83-99

49 La Gerche A, Roberts T]. Straining the RV to predict the future. JACC Cardiovasc Imaging 2015; 8: 170-171

50 Mohammed SF, Hussain I, AbouEzzeddine OF et al. Right ventricular function in heart failure with preserved ejection fraction: a community-based study. Circulation 2014; 130: 2310-2320

51 Guazzi M, Naeije R, Arena R et al. Echocardiography of right ventriculoarterial coupling combined with cardiopulmonary exercise testing to predict outcome in heart failure. CHEST 2015; 148 : 226-234

52 Kusunose K, Popović ZB, Motoki H, Marwick TH. Prognostic significance of exercise-induced right ventricular dysfunction in asymptomatic degenerative mitral regurgitation. Circ Cardiovasc Imaging 2013; 6: 167-176

53 Lancellotti P, Magne J, Dulgheru R et al. Clinical significance of exercise pulmonary hypertension in secondary mitral regurgitation. Am J Cardiol 2015; 115: 1454-1461
54 Meta-analysis Global Group in Chronic Heart Failure (MAGGIC). The survival of patients with heart failure with preserved or reduced left ventricular ejection fraction: an individual patient data meta-analysis. Eur Heart J 2012; 33: 1750-1757

55 Lam CSP, Roger VL, Rodeheffer RJ et al. Pulmonary hypertension in heart failure with preserved ejection fraction. J Am Coll Cardiol 2009; 53: 1119-1126

56 Hoendermis ES, Liu LCY, Hummel YM et al. Effects of sildenafil on invasive haemodynamics and exercise capacity in heart failure patients with preserved ejection fraction and pulmonary hypertension: a randomized controlled trial Eur Heart J 2015: ehv336

57 McMurray J], Adamopoulos S, Anker SD et al. ESC guidelines for the diagnosis and treatment of acute and chronic heart failure 2012: The Task Force for the Diagnosis and Treatment of Acute and Chronic Heart Failure 2012 of the European Society of Cardiology. Developed in collaboration with the Heart Failure Association (HFA) of the ESC. Eur J of Heart Fail 2012; 14: 803-869

58 Asgar AW, Mack M], Stone GW. Secondary mitra regurgitation in heart failure. J Am Coll Cardiol 2015; 65: 1231-1248

59 Gaemperli O, Moccetti M, Surder D et al. Acute haemodynamic changes after percutaneous mitral valve repair: relation to mid-term outcomes. Heart 2012; 98: 126-132

60 Upadhyay GA, Chatterjee NA, Kandala J et al. Assessing mitral regurgitation in the prediction of clinical outcome after cardiac resynchronization therapy. Heart Rhythm 2015; 12: 1201-1208

61 Malouf JF, Enriquez-Sarano M, Pellikka PA et al. Severe pulmonary hypertension in patients with severe aortic valve stenosis: clinical profile and prognostic implications. I Am Coll Cardiol 2002; 40: 789-795

62 Pai RG, Varadarajan P, Kapoor N, Bansal RC. Aortic valve replacement improves survival in severe aortic stenosis associated with severe pulmonary hypertension. Ann Thorac Surg 2007; 84: 80-85

63 Sinning JM, Hammerstingl C, Chin D et al. Decrease of pulmonary hypertension impacts on prognosis after transcatheter aortic valve replacement. Eurolntervention 2014; 9: 1042-1049

64 Maoqin S, Guoxiang H, Zhiyuan S et al. The clinical and hemodynamic results of mitral balloon valvuloplasty for patients with mitral stenosis complicated by severe pulmonary hypertension. Eur I Int Med 2005; 16: 413-418

65 Ward C, Hancock BW. Extreme pulmonary hypertension caused by mitral valve disease. Natural history and results of surgery. British Heart ] 1975; 37: 74-78

66 The Joint Task Force on the Management of Valvular Heart Disease of the European Society of Cardiology (ESC) and the European Association for Cardio-Thoracic Surgery (EACTS), Vahanian A Alfieri $\mathrm{O}$, Andreotti $\mathrm{F}$ et al. Guidelines on the management of valvular heart disease (version 2012). Eur Heart J 2012; 33: 2451-2496 
CME-Teilnahme

- Viel Erfolg bei Ihrer CME-Teilnahme unter http://cme.thieme.de

- Diese Fortbildungseinheit ist 12 Monate online für eine CME-Teilnahme verfügbar.

- Sollten Sie Fragen zur Online-Teilnahme haben, unter http:// cme.thieme.de/hilfe finden Sie eine ausführliche Anleitung.

1. Welche Aussage trifft zu?

a. Endothelin führt über eine Vasodilatation zur Verringerung des pulmonalvaskulären Widerstands (PVR).

b. Eine akute Erhöhung des PVR durch subtotale Verlegung der Lungenstrombahn wird von herzgesunden Menschen i.d.R. problemlos kompensiert.

c. Für den Großteil der PH-Patienten (pulmonale Hypertonie) ist eine medikamentöse PVR-Senkung grundlegendes Therapiekonzept.

d. Die pulmonale Hypertonie ist meist Folge einer LV-Dysfunktion.

e. In großen Höhen kommt es durch den verringerten Sauerstoffpartialdruck der Umgebungsluft zu einem Abfall des PAP.

2. Welche Aussage trifft nicht zu?

a. COPD-Patienten entwickeln typischerweise ein Cor pulmonale.

b. Systemische Entzündungsprozesse bei chronischen Lungenerkrankungen (CLE) begünstigen die Entstehung von Atherosklerose.

c. Die Entwicklung einer PH ist bei CLE prognostisch ungünstig.

d. Eine Langzeitsauerstofftherapie kann bei einer PH im Rahmen einer COPD die Prognose verbessern.

e. Das kardiovaskuläre Risiko von Patienten mit COPD oder idiopathischer Lungenfibrose ist gegenüber der Normalbevölkerung erhöht.

3. Welche Aussage trifft zu?

a. Bei Patienten mit Dyspnoe spricht eine Hyperkapnie gegen eine akute Linksherzinsuffizienz.

b. Eine unauffällige Bodyplethysmographie spricht gegen eine PH.

c. Die Echokardiografie kann indirekte Hinweise auf einen erhöhten PAP liefern.

d. Ein Rechtsherzkatheter ist zur Klassifizierung einer PH bei kardiopulmonal komorbiden Patienten verzichtbar.

e. Bei der COPD liegt typischerweise eine isolierte Einschränkung der Diffusionskapazität vor.

4. Welche Aussage trifft zu?

a. ACE-Hemmer, Betablocker und Aldosteronantagonisten bilden die Grundlage der Therapie der Rechtsherzinsuffizienz.

b. Bei terminaler Rechtsherzinsuffizienz bei PH sollte die Indikation zur Herz-Lungentransplantation geprüft werden.

c. Die Rechtsherzinsuffizienz bei postkapillärer $\mathrm{PH}$ wird als Cor pulmonale bezeichnet.

d. Eine RV-Dysfunktion hat auf die Prognose von Linksherzerkrankungen keinen wesentlichen Einfluss.

e. Typisches radiologisches Zeichen der Rechtsherzinsuffizienz ist die pulmonalvenöse Stauung.

5. Welche Aussage trifft zu?

a. Eine Operation stellt bei der CTEPH die Ultima ratio dar.

b. Prostazyklinanaloga eignen sich ausschließlich für die orale Therapie.

c. Die Gabe von PDE-5-Inhibitoren (Z.B. Sildenafil) bei PH im Rahmen von Linksherz- oder Lungenerkrankungen ist ein Off -Label-Einsatz.

d. Einzige bei CTEPH zugelassene Substanzklasse sind die Endothelinrezeptorantagonisten (z.B. Bosentan).

e. Bei allen PH-Formen ist eine therapeutische Antikoagulation indiziert.

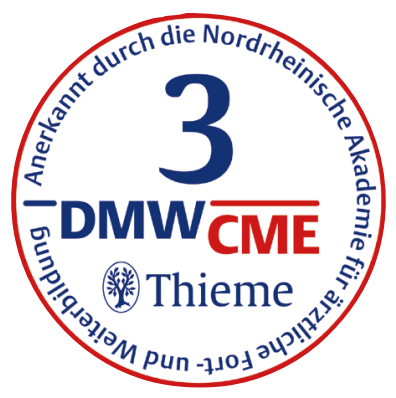

6. Welche Aussage trifft zu?

a. Zentrales Schlaf-Apnoe-Syndrom(CSA) und systolische Herzinsuffizienz treten selten zugleich auf.

b. Das obstruktive Schlaf-Apnoe-Syndrom sollte primär mittels adaptiver Servoventilation (ASV) behandelt werden.

c. Bei unbehandeltem OSA kommt es typischerweise rasch zu einer PH mit Rechtsherzinsuffizienz.

d. Patienten mit schlafbezogenen Atmungsstörungen haben ein verringertes kardiovaskuläres Risiko.

e. Patienten mit systolischer Herzinsuffizienz und CSA profitieren nicht von einer ASV.

7. Welche Aussage trifft zu?

a. Aufgrund der hohen Inzidenz der Lungenarterienembolie (LAE) ist die CTEPH eine der häufigsten Ursachen der pulmonalen Hypertonie.

b. Echokardiografie und myokardiale Nekroseparameter haben keinen Stellenwert in der Risikostratifizierung der LAE.

c. Neu aufgetretene Rechtsherzbelastungszeichen im EKG sprechen gegen eine akute Lungenarterienembolie.

d. Die Prognose der LAE wird nicht in erster Linie durch das Ausmaß der Oxygenierungsstörung bestimmt.

e. Die LV-Funktion wird auch im Rahmen schwerer LAE nicht relevant beeinträchtigt.

8. Welche Aussage trifft nicht zu?

a. Bei der chronischen Herzinsuffizienz liegt in 20-31\% der Fälle auch eine COPD vor.

b. Bei der COPD liegt in $20 \%$ der Fälle auch eine chronische Herzinsuffizienz vor.

c. Bei COPD sind $\beta$-Blocker wegen der Gefahr der Bronchokonstriktion kontraindiziert.

d. Eine systemische Inflammation liegt u.a. der häufigen Komorbidität von COPD und KHK zugrunde.

e. $\quad \beta_{2}$-Mimetika führen bei der COPD zu vermehrten Arrhythmien und anderen kardiovaskulären Ereignissen.

9. Welche Aussage trifft zu?

a. Eine pulmonale Hypertonie im Rahmen einer asymptomatischen Aortenklappeninsuffizienz bedeutet auch bei erhaltener systolischer LV-Funktion eine OP-Indikation.

b. Die Inzidenz des Eisenmenger-Syndroms ist in den letzten Jahren zunehmend.

c. Mitra-Clipping kann die postkapilläre PH bei Mitralklappeninsuffizienz günstig beeinflussen.

d. Das Ausmaß der postkapillären PH im Rahmen einer Aortenklappenstenose ist eher vom Schweregrad der systolischen als der diastolischen LV-Dysfunktion abhängig.

e. Das pulmonalvaskuläre Remodeling bei kongenitalen Vitien kann heute medikamentös unterbunden werden.

10. Welche Aussage trifft zu?

a. Der proinflammatorische Effekt von Tabakrauch ist auf das Atemwegsepithel beschränkt.

b. $\quad \beta_{2}$-Mimetika verbessern bei COPD die Prognose.

c. Inhalative Kortikosteroide führen bei der COPD häufig zu kardiovaskulären Komplikationen.

d. Patienten mit fortgeschrittener COPD, die das Rauchen aufgegeben haben, weisen kein erhöhtes kardiovaskuläres Risiko auf.

e. Substanzen, die das Auftreten kardiovaskulärer Ereignisse reduzieren, verbessern die Prognose bei COPD. 


\section{Angaben zur Person}

Name, Vorname, Titel:

Straße, Hausnr.:

PLZ, Ort:

Anschrift: $\square$ privat $\square$ dienstlich

Ich bin Mitglied der Ärztekammer (bitte Namen der Kammer eintragen):

Jahr meiner Approbation:

Ich befinde mich in der Weiterbildung zum:

Ich habe eine abgeschlossene Weiterbildung in ...

(bitte Fach eintragen):

Ich bin tätig als: $\square$ Assistenzarzt $\square$ Oberarzt $\square$ Chefarzt $\square$ niedergelassener Arzt $\square$ Sonstiges

Ich bin DMW-Abonnent: $\square$ ja $\square$ nein

Falls nein: ich habe den Fragebogen aus/von:

$\square$ Thieme-connect $\square$ Kollegen $\square$ der Klinik $\square$ einer Bibliothek

$\square$ Sonstiges

Lernerfolgskontrolle (Eine Antwort pro Frage ankreuzen)
1. $\square$ A $\square$ B $\square$ C $\square$ D $\square$ E
2. $\square$ A $\square$ B $\square \mathrm{C} \quad \square$ D $\square$ E
3. $\square \mathrm{A} \quad \square \mathrm{B} \quad \square \mathrm{C} \quad \square \mathrm{D} \quad \square \mathrm{E}$
4. $\square$ A $\square$ B $\square$ C $\square$ D $\square$ E
5. $\square \mathrm{A} \quad \square \mathrm{B} \quad \square \mathrm{C} \quad \square \mathrm{D} \quad \square \mathrm{E}$
6. $\square$ A $\square$ B $\square$ C $\square$ D $\square \mathrm{E}$
7. $\square \mathrm{A} \quad \square \mathrm{B} \quad \square \mathrm{C} \quad \square \mathrm{D} \quad \square \mathrm{E}$
8. $\square$ A $\square$ B $\square$ C $\square$ D $\square$ E
9. $\square \mathrm{A} \square \mathrm{B} \quad \square \mathrm{C} \quad \square \mathrm{D} \quad \square \mathrm{E}$
10. $\square \mathrm{A} \quad \square$ B $\square \mathrm{C} \quad \square \mathrm{D} \quad \square \mathrm{E}$

Ich versichere, dass ich die Beantwortung der Fragen selbst und ohne fremde Hilfe durchgeführt habe

Ort, Datum:

Unterschrift:

Bitte in dieses Feld Ihre DMW Abonnement-Nummer eintragen:

\section{Fragen zur Zertifizierung}

1. Das Thema des Beitrages kommt in meiner ärztlichen Tätigkeit $\square$ häufig vor $\square$ selten vor $\square$ regelmäßig vor $\square$ gar nicht vor

2. Bei diesem Thema habe ich $\square$ eine feste Gesamtstrategie $\square$ keine Strategie $\square$ noch offene Einzelprobleme

3. In Bezug auf das Thema des Beitrages

$\square$ fühle ich mich nach dem Studium des Beitrags in meiner Strategie bestätigt

$\square$ habe ich meine Strategie verändert:

$\square$ habe ich erstmals eine einheitliche Strategie erarbeitet

$\square$ habe ich keine einheitliche Strategie ableiten können

4. Wurden aus der Sicht Ihrer täglichen Praxis heraus wichtige Aspekte des Themas

nicht erwähnt: $\square$ ja, welche $\square$ nein

zu knapp abgehandelt? $\square$ ja, welche $\square$ nein

überbewertet? $\square$ ja, welche $\square$ nein

5. Verständlichkeit des Beitrages

$\square$ Der Beitrag ist nur für Spezialisten verständlich

$\square$ Der Beitrag ist auch für Nicht-Spezialisten verständlich

6. Beantwortung der Fragen

$\square$ Die Fragen lassen sich aus dem Studium des Beitrags allein beantworten

$\square$ Die Fragen lassen sich nur unter Zuhilfenahme zusätzlicher Literatur beantworten

7. Die Aussagen des Beitrages benötigen eine ausführlichere Darstellung

$\square$ zusätzlicher Daten

$\square$ von Befunden bildgebender Verfahren

$\square$ die Darstellung ist ausreichend

8. Wieviel Zeit haben Sie für das Lesen des Beitrages und der Bearbeitung des Quiz benötigt?

Zertifizierungsfeld (wird durch die DMW ausgefülltt)

Ihr Ergebnis:

Sie haben

von 10 Fragen richtig beantwortet.

Sie haben $\square$ bestanden und 3 Punkte erworben

$\square$ nicht bestanden $\square$ ungültig, weil:

Stuttgart, den Stempel/Unterschrift 